\title{
Academic Hospital Medicine 2.0: If You Aren't Teaching Residents, Are You Still Academic?
}

\author{
Carrie A Herzke, MD, MBA*, Daniel P Hunt, MD², Daniel J Brotman, MD
}

'Department of Medicine, Johns Hopkins University, Baltimore, Maryland; '²Department of Medicine, Emory University, Atlanta, Georgia.

ropons

ow much teaching time will I get in my first year on faculty?" Leaders at academic hospitalist programs know to expect this question from almost every applicant. We also know that we will be graded on our response; the more resident-covered service time, the better. For some applicants, this question is a key litmus test. Some prospective faculty choose to pursue academic hospital medicine because of their own experiences on the wards during residency. They recall the excitement of leading a team of interns and students under the wing of a seasoned attending, replete with chalk talks, clinical pearls, and inspired learners. Teaching time is more quantifiable than mentorship quality and academic opportunity, more important than salary and patient load for some, and more familiar than relative value unit expectations.

Over the past two decades, academic hospitalist programs have steadily grown, ${ }^{1}$ but their teaching footprints have not. ${ }^{2,3}$ Although historically some academic hospitalists spent almost $100 \%$ of their clinical time on teaching services, work hour rules and diversification of resident clinical time toward outpatient and subspecialty activities have decreased the amount of general medicine ward time for residents. ${ }^{2}$ In addition, as academic medical centers broadened their clinical networks, inpatient volumes exceeded the capacity of teaching services. Finally, several large academic medical centers and healthcare networks are acquiring or building additional hospitals, increasing the number of medical beds that are staffed by hospitalists without residents. ${ }^{4}$

In our experience, as academic healthcare systems continue to grow and hospital medicine programs rapidly expand to meet clinical needs, the percentage of clinical time spent on a traditional ward teaching service continues to decrease. In several academic hospitalist programs, the majority of faculty effort is now devoted to direct care, ${ }^{5}$ with limited residentcovered ward time spread across a larger group of faculty. The 2018 State of Hospital Medicine Report suggests that our experience is not unique, with academic programs caring for adults reporting that $31 \%$ of clinical work was on traditional ward teaching services, $16 \%$ on direct care services with intermittent learners, and $53 \%$ on nonteaching services. ${ }^{5}$

"Corresponding Author: Carrie Herzke, MD, MBA; Email: cherzke1@jhmi.edu; Telephone: (443) 287-3631

Published online first February 19, 2020.

Received: July 18, 2019; Revised: November 5, 2019;

Accepted: November 5, 2019

(c) 2020 Society of Hospital Medicine DOI 10.12788/jhm.3354
This current state of affairs raises a number of questions as follows:

- How can hospitalist program leaders take advantage of existing resident teaching opportunities?

- How should those teaching opportunities be allocated?

- What nontraditional teaching venues exist in academic medicine?

- How can faculty develop their teaching skills in an environment with limited traditional ward teaching time?

We believe that these changes require us to redefine what it means to be an academic hospitalist, both for existing faculty and for prospective faculty whose views of academic hospital medicine may have been shaped by role models seen only in their clinical teaching role.

\section{MAXIMIZING RESIDENT TEACHING OPPORTUNITIES}

Is reduced teaching time the new normal or will the pendulum swing back toward more resident teaching time for academic hospitalists? The former is likely the case. None of the current trends in medical education point to an expansion of residents in the inpatient setting. Although there may be some opportunities to assume general medicine attending time is currently covered by primary care physicians and subspecialists, in several programs, hospitalists already cover the overwhelming majority of general medicine teaching services.

Although there may be occasional opportunities for academic hospitalist programs to develop new teaching roles with residents or fellows (for example, by expanding to community sites with residency programs or to subspecialty teaching services, or by creating hospital medicine fellowships and resident or student electives), the reality is that we as hospitalists will need to adapt to direct care as the plurality of our work.

\section{ALLOCATING TEACHING TIME}

How should we allocate traditional teaching time among our faculty? Since it is a coveted-but relatively scarce-resource, teaching time should be allocated thoughtfully. Based on our collective experience, academic hospitalist groups have taken a variety of approaches to this challenge, including forming separate clinical groups at the same institution (a teaching faculty group and a nonteaching group), ${ }^{6}$ requiring all hospitalists to do some amount of direct care to facilitate distribution of teaching time or having merit or seniority-based teaching time allocation (based on teaching evaluations, formal teaching roles such as program director status, or years on faculty). 
Each approach to assigning teaching time has its challenges. Hospitalist leaders must manage these issues through transparency about the selection process for teaching rotations and open discussion of teaching evaluations with faculty. It is also critical that the recruitment process set appropriate expectations for faculty candidates. Highlighting academic opportunities outside of teaching residents, including leadership roles, quality improvement work, and research, may encourage applicants and current hospitalists to explore more varied career trajectories. Hospitalists focusing on these other paths may elect to have less teaching time, freeing up opportunities for dedicated clinician educators.

\section{BEYOND TRADITIONAL RESIDENT TEACHING TEAMS}

What other ward-based teaching opportunities might be available for academic hospitalists who do not have the opportunity to attend on traditional resident teaching teams? As supervisory requirements for residents have been strengthened, expansion of teaching into the evening and overnight hours to supervise new admissions to the teaching services has been one approach to augment teaching footprints. ${ }^{7,8}$

In addition, nontraditional teaching teams such as attending/intern teams (without a supervising resident) or attending/ subintern (fourth-year medical student) teams have been developed at some institutions. ${ }^{9}$ Although allowing for additional exposure to learners, these models require a more hands-on approach than traditional teaching teams, particularly at the start of the academic year. Finally, as hospitalist teams have grown to include advanced practice providers (APPs), some programs have established formal teaching programs to address professional development needs of these healthcare professionals. ${ }^{10,11}$

\section{DEVELOPING HOSPITALIST EDUCATORS}

How do we help junior faculty who have the potential to be talented educators succeed in teaching when they have limited opportunities to engage with residents on clinical services? One approach is to encourage hospitalists to participate in resident didactic sessions such as "morning report" and noon conference. Another approach is to focus on teaching other learners. For example, several academic medical centers provide opportunities for hospitalists to engage in student teaching, either on the wards or via classroom instruction. In addition, as mentioned previously, APPs who are new to hospital medicine are an engaged audience and represent an opportunity for hospitalist educators to utilize and hone their teaching skills. Finally, organizing lectures for nursing colleagues is another way for the faculty to practice "chalk talks" and develop teaching portfolios.

Hospitalists can also leverage their expertise to build systems in which academic hospitalists are teaching each other, creating a culture of continuous learning. These activities may include case conferences, morbidity and mortality conferences, journal clubs, clinical topic updates developed by and for hospitalists, simulation exercises, and other group learning sessions. Giving hospitalists the opportunity to teach each other allows for professional growth that is not dependent on the presence of traditional learners.

\section{REDEFINING ACADEMIC HOSPITALISTS}

Philosophically, a key question is "What makes 'academic' academic?" Traditionally, academic hospitalist positions were synonymous with resident teaching or, for a small number of academic hospitalists, significant funded research. In an era in which teaching residents may no longer be part of the job description for many hospitalists at academic medical centers, what distinguishes these positions from $100 \%$ clinical positions and what are the implications for academic hospital medicine?

Although data regarding why hospitalists seek "nonteaching" positions at academic medical centers are lacking, we believe that these jobs remain popular due to opportunities that are perceived to be unique to academic medical centers. These include more flexible scheduling (academic programs may be less likely to have seven-on/seven-off schedules), exposure to research and cutting-edge technology, opportunities to care for tertiary and quaternary care patients, collaboration with academic peers and experts in the field, and interaction with a range of learners, including medical, pharmacy, advanced practitioner, and other students.

Understanding the motivation of candidates who apply for academic hospital medicine positions_-aside from supervising/ teaching residents-will be an important goal for academic hospitalist leaders to ensure future success in staffing growing programs and creating sustainable academic hospitalist careers. As resident teaching time decreases, implementing robust faculty or professional development programs to address the broader interests and needs of academic hospitalist faculty will be increasingly important. Sehgal et al described one such program for faculty development, ${ }^{12}$ and a more recent paper outlines a faculty development program focused on quality improvement and patient safety. ${ }^{13}$ These types of programs provide opportunities for academic hospitalists to engage in academic pursuits that are independent of residency programs.

\section{CONCLUSION}

Therefore, what do we tell the eager faculty applicant? First, we should not hide from the honest answer, ie, new faculty may not get as much resident teaching time as they would like or expect. Although we want hospitalists to maintain integral involvement in residency training programs, we also want to build a culture of clinical excellence, scholarship, and continuous learning that is not dependent on directly teaching residents. We should highlight the unique opportunities of academic hospital medicine, including teaching other learners, caring for tertiary/quaternary care patients, working with colleagues who are leaders in their field, and engaging in research and quality improvement work. By capitalizing on these opportunities, we can actively redefine what makes "academic" academic and ensure that we sustain academic hospital medicine as a desirable and rewarding career. 
Disclosures: The authors have nothing to disclose.

\section{References}

1. Wachter RM, Goldman L. Zero to 50,000-the 20th anniversary of the hospitalist. N Engl J Med 2016;375(11):1009-1011. https://doi.org/10.1056/NEJMp1607958

2. Saint $\mathrm{S}$, Flanders SA. Hospitalists in teaching hospitals: opportunities but not without danger. J Gen Intern Med. 2004;19(4):392-393. https://doi. org/10.1111/j.1525-1497.2004.42002.x

3. Flanders SA, Saint S, McMahon LF, Howell JD. Where should hospitalists sit within the academic medical center? J Gen Intern Med. 2008;23(8):12691272. https://doi.org/10.1007/s11606-008-0682-1

4. Paavola A. Five hospital projects announced this year worth $\$ 1 \mathrm{~B}$ or more. Becker's Hospital Review. July 1, 2019. Accessed August 24, 2019. https:// www.beckershospitalreview.com/capital/5-hospital-projects-announcedthis-year-worth-1b-or-more.html

5. White A, Anders J, Anoff DL, Creamer J, Flores LA. Table 3.45 Distribution of work in academic hmgs. In: 2018 State of Hospital Medicine (SoHM) Report. Society of Hospital Medicine; 2018.

6. Hunt D, Burger A, Harrison R, Southern W, Boonyasai RT, Leykum L. Hospitalist staffing: to split or not to split? SGIM Forum 2013;36:6.
7. Farnan JM, Burger A, Boonyasai RT, et al. Survey of overnight academic hospitalist supervision of trainees. J Hosp Med. 2012;7(7):521-523. https://doi. org/10.1002/jhm.1961

8. Sani SN, Wistar E, Le L, Chia D, Haber LA. Shining a light on overnight education: Hospitalist and resident impressions of the current state, barriers, and methods for improvement. Cureus 2018;10:e2939. https://doi.org/10.7759/cureus.2939

9. O'Leary KJ, Chadha V, Fleming VM, Martin GJ, Baker DW. Medical subinternship: student experience on a resident uncovered hospitalist service. Teach Learn Med. 2008;20(1):18-21. https://doi.org/10.1080/10401330701797974

10. Klimpl D, Franco T, Tackett $S$, et al. The current state of advanced practice provider fellowships in hospital medicine: a survey of program directors. $J$ Hosp Med. 2019;14(7):401-406. https://doi.org/10.12788/jhm.3191

11. Lackner $C$, Eid S, Panek T, Kisuule F. An advanced practice provider clinical fellowship as a pipeline to staffing a hospitalist program. J Hosp Med. 2019;14(6):336-339. https://doi.org/10.12788/jhm.3183

12. Sehgal NL, Sharpe BA, Auerbach AA et al. Investing in the future: building an academic hospitalist faculty development program. J Hosp Med. 2011;6(3):161-166. https://doi.org/10.1002/jhm.845

13. van Schaik SM, Chang A, Fogh $S$, et al. Jumpstarting faculty development in quality improvement and patient safety education: A team-based approach. Acad Med. 2019;94(11):1728-1732. https://doi.org/10.1097/ ACM.00000000000002784 